\title{
Conceitos atuais sobre a intrusão traumática de dentes per- manentes: epidemiologia, efeitos de fatores antes e durante o trauma e variáveis de tratamento
}

\author{
Flavia Artese*
}

Recentemente, Andreasen e sua equipe publicaram uma trilogia de $\operatorname{artigos}^{1,2,3}$, baseados em uma análise longitudinal dos casos de intrusão de dentes permanentes atendidos no serviço de trauma do Hospital Universitário de Copenhagen, acompanhados de 1955 a 2003, totalizando 216 dentes. Questões epidemiológicas, fatores antes e durante o trauma e variações de tratamento que afetariam a recuperação de um dente intruído foram abordados.

No primeiro artigo os autores apresentam de maneira detalhada o protocolo utilizado para obter informações clínicas, documentação fotográfica e radiográfica de pacientes que sofreram trauma dentário no serviço do Hospital Universitário de Copenhagen. Após colherem estes dados, os pacientes com dentes intruídos eram submetidos a um destes três métodos de tratamento: aguardar erupção espontânea, reposicionamento cirúrgico ou extrusão ortodôntica. Além disso todos os dentes tratados eram esplintados por 6 a 8 semanas e $500 \mathrm{mg}$ de penicilina eram administrados 4 vezes ao dia durante 4 dias. Os pacientes eram acompanhados em intervalos regulares de 3, 4, 6 e 8 semanas, 6 meses e anualmente até 5 anos após o trauma. Os resultados deste estudo epidemiológico mostram que a intrusão constitui $1,9 \%$ dos traumas em dentes permanentes, sendo sua causa principal a queda contra superfícies duras. A faixa etária entre 6 e 12 anos foi a mais acometida, ocorrendo mais cedo com os meninos do que com as meninas. $\mathrm{O}$ padrão de trauma poderia incluir de $1 \mathrm{a}$ 5 dentes, sendo mais comum 1 ou 2 dentes intruídos. A localização mais freqüente é na região anterior da maxila, sendo muito rara nos dentes inferiores. A extensão de intrusão pode variar de $1 \mathrm{a} 20 \mathrm{~mm}$, sendo mais comum até $8 \mathrm{~mm}$. O parâmetro clínico mais usual foi o som oco à percussão (79\%) e o parâmetro radiográfico mais confiável para avaliar a quantidade de intrusão foi a distância da junção amelo-cementária de um dente irrompido ao do dente intruído. A obliteração do ligamento periodontal foi vista em $51,8 \%$ dos casos.

O segundo artigo teve como objetivo avaliar os efeitos de fatores existentes antes e durante o trauma sobre possíveis complicações pós-tratamento, isto é, a necrose pulpar (NP), reabsorção radicular (RR) e perda de inserção periodontal (PP). Os fatores considerados foram: gênero, idade, estágio de desenvolvimento radicular, localização do dente, fraturas dentárias associadas, se- veridade do deslocamento e o número de dentes envolvidos. Dos 216 dentes avaliados inicialmente 76 foram retirados do estudo, totalizando 140 dentes. Os resultados apresentaram que 124 dentes sofreram NP, sendo a maior parte em 6 meses pós-trauma. A NP estava significativamente associada ao grau de desenvolvimento radicular, sendo mais tardia em dentes com rizogênese incompleta. A RR ocorreu em 67 casos e também estava associada com o grau de desenvolvimento radicular. A maior parte dos casos foi diagnosticada 1 ano após o trauma, apresentando um platô de cicatrização nos 5 anos subseqüentes e havendo reinício da reabsorção até 10 anos após o trauma. A PP ocorreu em 45 casos e novamente estava associada com o grau de desenvolvimento radicular. A maior parte da perda de inserção ocorreu nos primeiros 4 anos em casos com rizogênese incompleta, enquanto dentes com raízes completamente formadas apresentavam perda de inserção contínua, durante todo o período de observação. Quando estes dados foram analisados com as variáveis antes e durante o trauma, verificou-se que quanto maior a idade, maior o número de complicações pós-tratamento. A mesma situação foi verificada com o grau de desenvolvimento radicular. A PP está mais associada com a intrusão dos incisivos laterais. Nos casos de fraturas coronárias existe associação com NP. O grau de severidade da intrusão, no caso, mais do que $7 \mathrm{~mm}$, aumenta a chance de haver todas as complicações consideradas (NP, RR e PP). O número de dentes intruídos aumenta a chance de PP, principalmente nas regiões proximais entre dois dentes adjacentes traumatizados.

O terceiro artigo propôs avaliar a influência de fatores de tratamento como demora no atendimento, método de reposicionamento, tipo de esplintes, tempo de esplintagem e o uso de antibióticos, sobre as mesmas complicações pós-tratamento (NP, RR e PP). A mesma amostra de 140 dentes foi utilizada. Os métodos de esplintagem utilizados foram: rígida (esplintes feitos de prata ou resina acrílica), semi-rígida (com fios ortodônticos) e flexível (esplintes em resina flexível). Os métodos de reposição do dente intruído foram re-erupção espontânea, reposicionamento cirúrgico ou extrusão ortodôntica. Nos casos que foram atendidos um dia após o trauma apenas a PP teve pior resultado. Os dentes que não foram reposicionados, isto é, onde aguardou-se a re-erupção espontânea, apresentaram melho- 
res chances para evitar a NP, RR e PP, principalmente nos casos com rizogênese incompleta. Acredita-se que o tracionamento ortodôntico ou o reposicionamento cirúrgico de dentes intruídos, durante o processo de cicatrização, possam ser considerados um "novo trauma", alterando os processos celulares e portanto, obtendo-se piores resultados do que ao apenas aguardar a re-erupção espontânea. Geralmente, a re-erupção espontânea ocorreu entre 2 e 13 meses do trauma. O tipo de esplintagem não apresentou diferenças sobre o resultado do tratamento, assim como o tempo de esplintagem ou o uso de antibióticos.

A intrusão de dentes é bastante rara, constituindo apenas 1,9\% dos traumas em dentes permanentes. Esta ocorrência escassa faz com que poucos dados sobre este assunto estejam disponíveis na literatura odontológica e com que este estudo seja de grande validade clínica. O acompanhamento longitudinal destes tratamentos é uma das principais formas para se chegar a protocolos clínicos. Porém, com novas técnicas nas áreas de imagem, como por exemplo a tomografia odontológica, e com métodos bioquímicos, o acompanhamento destes casos de trauma poderão elucidar questões ainda não resolvidas e permitir que a Odontologia moderna salve ainda mais dentes traumatizados.

1. ANDREASEN, J. O.; BAKLAND, L. K.; MATRAS, R. C.; ANDREASEN, F. M. Traumatic intrusion of permanent teeth. Part 1. An epidemiological study of 216 intruded permanent teeth. Dent Traumatol, Copenhagen, v. 22, n. 2, p. 83-89, April 2006.

2. ANDREASEN, J. O.; BAKLAND, L. K.; ANDREASEN, F. M. Traumatic intrusion of permanent teeth. Part 2. A clinical study of the effects of preinjury and injury factors, such as sex, age, stage of root development, tooth location, and extent of injury, including number of intruded teeth no 140 intruded permanent teeth. Dent Traumatol, Copenhagen, v. 22, n. 2, p. 90-98, April 2006.

3. ANDREASEN, J. O.; BAKLAND, L. K.; ANDREASEN, F. M. Traumatic intrusion of permanent teeth. Part 3. A clinical study of the effect of treatment variables such as treatment delay, method of repositioning, type of splint, length of splinting and antibiotics on 140 teeth. Dent Traumatol, Copenhagen, v. 22, n. 2, p. 99111, April 2006.

\section{Marcadores biológicos para a avaliação de reabsorção radicular}

A identificação precoce da predisposição para reabsorção radicular severa ao início do tratamento ortodôntico seria fundamental para evitar graves seqüelas às raízes dentárias. A dentina é depositada continuamente ao longo da vida na superfície pulpar e contém algumas proteínas específicas como a proteína 1 da matriz dentinária (DMP1), a fosfoforina dentinária (PP) e a sialoproteína dentinária (DSP). Estas proteínas de matriz extracelular, que estão associadas unicamente à mineralização da dentina sobre a superfície pulpar, não são liberadas no fluido gengival crevicular (FGC), a não ser que esteja havendo exposição desta superfície em decorrência da reabsorção radicular. A quantificação e identificação das proteínas liberadas no FGC poderia permitir a avaliação do grau de reabsorção radicular de maneira precoce, logo ao início do tratamento ortodôntico.

Com este intuito, 60 pacientes do Departamento de Ortodontia da Universidade de Illinois, EUA, foram selecionados e dividos em três grupos $(n=20)$. O primeiro grupo incluiu pacientes em tratamento ortodôntico com reabsorção radicular suave $(<2 \mathrm{~mm})$, o outro incluiu pacientes com reabsorção radicular severa ( $>2 \mathrm{~mm}$ ), e o grupo controle consistia de pacientes que ainda não haviam iniciado o tratamento ortodôntico. Coletou-se o FGC de todos os pacientes, que foi analisado através de eletroforese em gel de SDS-policacrilamida (SDS-PAGE), Western blotting e ELISA. Na análise por SDS-PAGE, que separa proteínas de acordo com seu peso molecular, verificou-se maior concentra- ção de proteínas nos grupos com reabsorção radicular. A identificação das proteínas de dentina em questão, separadas pelo SDS-PAGE, foi feita pelos métodos de Western blotting e ELISA, os quais mostraram que, em ambos os testes, havia maior quantidade de DMP1, PP e DSP nos grupos com reabsorção radicular. Desta forma, estas proteínas poderiam servir como marcadores biológicos para a determinação da reabsorção radicular.

O aprimoramento desta técnica permitiria desenvolver testes clínicos, que poderiam ser usados ao início do tratamento ortodôntico, obtendo-se, de maneira precisa o grau de predisposição para reabsorção radicular de um paciente no primeiro mês de tratamento, o que não é possivel com os métodos de imagens atuais. Esta informação interferiria de maneira positiva nos planos de tratamento, fazendo com que o clínico buscasse terapias mais curtas e utilizasse forças leves ao longo de toda a intervenção ortodôntica. Um exemplo simples seria na situação de casos limítrofes, onde a predisposição para reabsorção radicular poderia pesar contra as extrações de dentes, na busca de um menor tempo de tratamento.

1. BALDUCCI, L. A.; RAMACHANDRAN, A.; HAO, J.; NARAYANAN, K.; EVANS, C.; GEORGE, A. Biological markers for evaluation of root resorption. Arch Oral Biol, Exeter, v. 52, p. 203-208, Jan. 2007.

* Professora Adjunta de Ortodontia da Universidade do Estado do Rio de Janeiro. Mestre e Doutora em Ortodontia pela Universidade Federal do Rio de Janeiro. Ortodontista diplomada pelo Board Brasileiro de Ortodontia e Ortopedia Facial. 